\title{
Near-Capacity \\ Irregular-Convolutional-Coding-Aided Irregular Precoded Linear Dispersion Codes
}

\author{
Nan Wu and Lajos Hanzo, Fellow, IEEE
}

\begin{abstract}
In this paper, we propose novel serial concatenated irregular convolutional-coded (IRCC) irregular precoded linear dispersion codes (IR-PLDCs), which are capable of operating near the multiple-input-multiple output (MIMO) channel's capacity. The irregular structure facilitates the proposed system's nearcapacity operation across a wide range of SNRs, while maintaining a vanishing bit error ratio (BER). Each coding block of the proposed scheme and all the iterative decoding parameters are designed with near-capacity operation in mind, using extrinsic information transfer charts. By applying the irregular design principle to both the inner and outer codes, the proposed IRCC-aided IR-PLDC scheme becomes capable of operating as close as $0.9 \mathrm{~dB}$ to the MIMO channel's capacity for SNRs in excess of a certain threshold.
\end{abstract}

Index Terms-EXIT-chart matching, EXIT-charts, irregular channel coding, irregular space-time coding, linear dispersion coding, near-capacity channel coding, wireless multiple-inputmultiple-output (MIMO) channel capacity.

\section{INTRODUCTION}

W IRELESS communication systems using multiple antennas at both the transmitter and receiver, which are referred to as multiple-input-multiple-output (MIMO) systems in the literature, have the potential of maintaining reliable transmissions at high data rates [1]-[3]. The design of coding schemes for MIMO systems operating at high SNRs involves a tradeoff between the achievable rate at which the system's capacity increases and the rate at which the error probability decays [4]. There is considerable interest in developing schemes that provide different tradeoffs in terms of the achievable rate and error probability, which are applicable for employment in a broad range of antenna configurations.

The set of linear dispersion codes (LDCs), which was first proposed by Hassibi and Hochwald [5], constitutes a wide-ranging class of space-time codes exhibiting diverse characteristics. Hence, this family encompasses numerous

Manuscript received October 23, 2007; revised August 23, 2008, October 21, 2008, and November 30, 2008. First published December 12, 2008; current version published May 29, 2009. This work was supported in part by the Engineering and Physical Sciences Research Council, U.K., and in part by the European Union under the Optimix project nINFSO-ICT-214625. The review of this paper was coordinated by Prof. E. Bonek.

The authors are with the School of Electronics and Computer Science, University of Southampton, SO171BJ Southampton, U.K. (e-mail: 1h@ecs. soton.ac.uk).

Color versions of one or more of the figures in this paper are available online at http://ieeexplore.ieee.org.

Digital Object Identifier 10.1109/TVT.2008.2010979 existing schemes [6], [7], providing a natural framework in which such design problems can be posed. The joint design of the space-time signal of several antennas using sphere-packing modulation and iterative detection was proposed in [8] and [9]. The revolutionary concept of LDCs [5], [10] invokes a matrix-based linear modulation framework, where each spacetime transmission matrix is generated by a linear combination of so-called "dispersion" matrices used to disperse or map the symbols to the transmit antennas, where the weights of the constituent matrices are determined by the transmitted symbols. The set of dispersion matrices can be optimized according to different objectives. The dispersion matrices were originally [5] designed for maximizing the continuousinput-continuous-output memoryless channel capacity [2] of the MIMO system. However, the LDCs proposed in [5] did not necessarily guarantee a low bit error ratio (BER) [10], [11]. On the other hand, LDCs can also be optimized using the determinant criterion [12] using the beneficial techniques of the Golden codes [13], [14], where a nonvanishing determinant is promised. By contrast, in this paper, we propose a novel method of optimizing the set of dispersion matrices for the sake of maximizing the discrete-input-continuous-output memoryless channel (DCMC) capacity.

Serial concatenated codes (SCCs) are capable of attaining a vanishing BER, while maintaining a manageable decoding complexity [15], [16]. It has been demonstrated in [17] and [18] that SCCs may operate near the MIMO channel's capacity at certain SNR values. However, the distance to the capacity is still quite significant, particularly when higher order modulation schemes are employed [17]. In [19] and [20], the authors proposed to adopt irregular convolutional codes (IRCCs) as the outer channel code of an SCC, since IRCCs exhibit flexible extrinsic information transfer (EXIT) chart characteristics [20]. Unfortunately, these schemes may fail to approach the capacity at different SNRs, where the inner and outer codes fail to create an open EXIT tunnel. Since LDCs have the ability to approach the MIMO channel's capacity and to provide flexible configurations, the novel contribution of this paper is the joint design of irregular LDCs as the inner code and the IRCCs as the outer code of an SCC scheme to approach the capacity for a wide SNR range.

More explicitly, motivated by the aforementioned flexibility of the irregular outer code design philosophy, in this paper, we circumvent the IRCC-related outer code limitations by proposing irregular precoded LDCs (IR-PLDCs) as the inner code of the SCCs and serially concatenate the resultant 


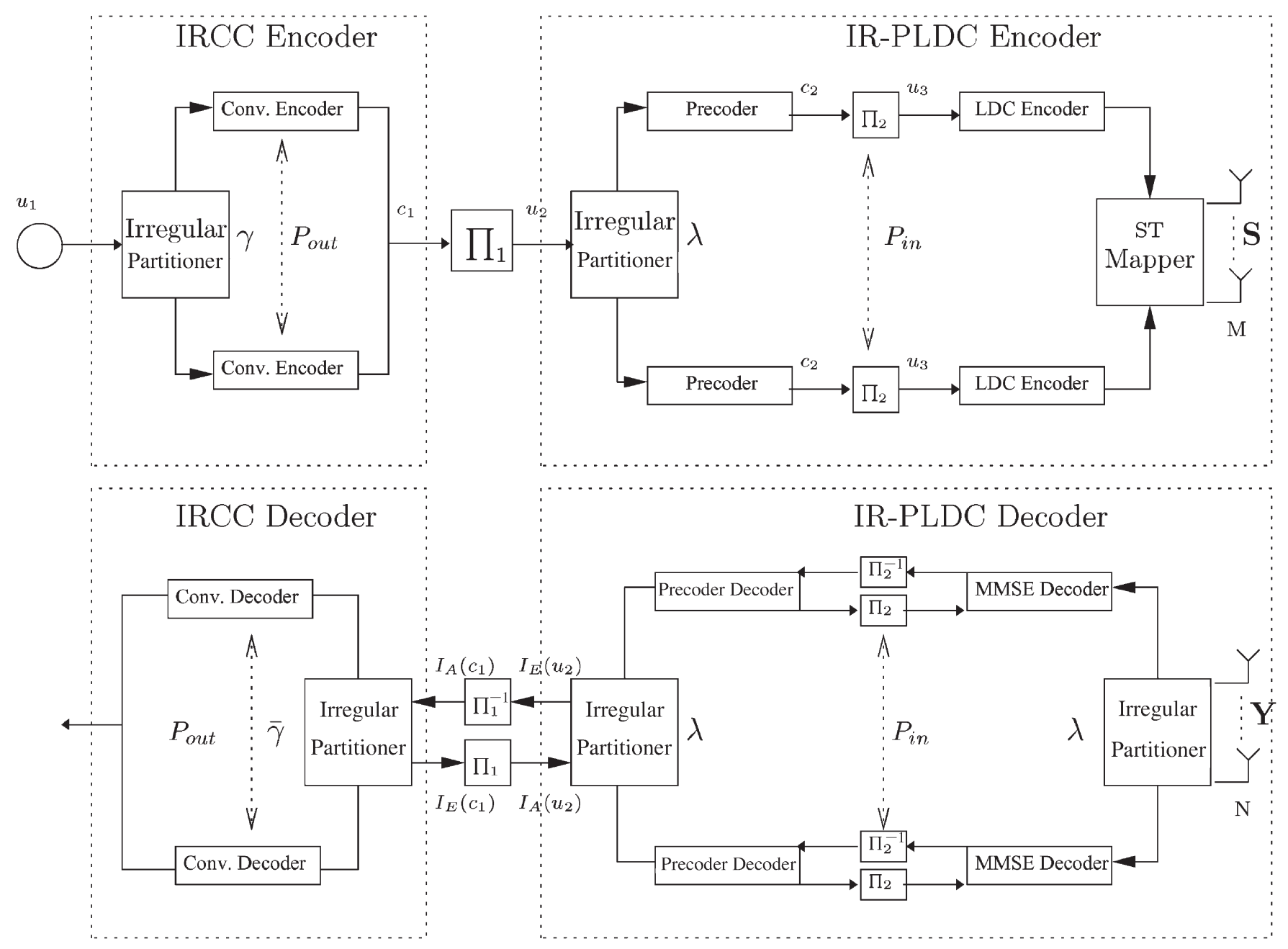

Fig. 1. Schema of the IRCC-coded IR-PLDC using iterative decoding.

IR-PLDCs with the outer IRCCs to operate close to the MIMO channel's capacity across a wide SNR region, while maintaining a vanishing BER. The rationale of using a unity-rate precoder in an SCC scheme [21] is that it allows us to create an infinite impulse response system at the cost of a low implementation complexity, which has the benefit of improving the extrinsic information exchange among the component codes at the receiver. The novelty of the proposed IRCC-coded IRPLDC scheme is described in the following.

- It is capable of operating close to the MIMO channel's capacity across a wide range of SNRs.

- The irregular design principle applied is to both the inner code using IR-PLDCs and the outer employing IRCCs.

- The constituent LDCs of the inner IR-PLDC scheme are optimized by maximizing the DCMC capacity.

- The maximum achievable rate of the IRCC-aided IRPLDC scheme is determined with the aid of EXIT charts [22], [23] when using minimum-mean-square-error (MMSE) detectors.

- The proposed scheme can be designed for an arbitrary number of transmit and receive antennas combined with arbitrary modulation schemes.

- IR-PLDCs may be reconfigured by activating different dispersion matrices for different IRCC component codes.
We commence our discourse by providing a detailed description of the proposed IRCC-coded IR-PLDC scheme's structure in Section II. In Section III, we demonstrate how the proposed system and all the iterative decoding parameters are optimized from a capacity maximization perspective. Our simulation results are discussed in Section IV. Finally, we conclude our discourse in Section V.

\section{System DESCRIPTION}

We consider a MIMO system employing $M$ transmit and $N$ receive antennas. Fig. 1 portrays the system model of the proposed serially concatenated IRCC-coded IR-PLDC scheme. A total of $P=P_{\text {out }}+P_{\text {in }}$ irregular components are employed in the proposed system, where the outer IRCC scheme contains $P_{\text {out }}$, while the inner IR-PLDC scheme has $P_{\text {in }}$ number of component codes.

At the transmitter in Fig. 1, a frame of information bits $u_{1}$ is encoded by an IRCC encoder. The particular fraction of the bitstream $u_{1}$, which will be fed into each IRCC component code, is controlled by the "irregular partitioner" in Fig. 1 based on the weighting coefficient vector $\gamma=\left[\gamma_{1}, \ldots, \gamma_{P_{\text {out }}}\right]$. The aggregate rate of the outer IRCC scheme is denoted as $R_{\text {out }}$, and the rate of its component codes is $R_{i, \mathrm{IRCC}}$, where $i=1, \ldots, P_{\text {out }}$. 
Then, the encoded bits $c_{1}$ are interleaved by a random bit interleaver, yielding the outer encoded bits $u_{2}$. Again, the IRPLDC's "irregular partitioner" feeds the appropriately selected fraction of $u_{2}$ into the various precoded LDC (PLDC) component codes, governed by the weighting coefficient vector $\boldsymbol{\lambda}=$ $\left[\lambda_{1}, \ldots, \lambda_{P_{\text {in }}}\right]$. Memory-1 unity-rate precoders were employed for all the PLDC components. We refer the readers to [21] for the encoding/decoding structure, as well as the calculation of the extrinsic information of the unity-rate precoders. Within each PLDC encoder, the resultant precoded bits $c_{2}$ are interleaved by a second interleaver, yielding the interleaved bits $u_{3}$, which are fed into the bit-to-symbol mapper of the LDC block in Fig. 1. After modulation, the information-bearing vector $\mathbf{K}=\left[s_{1}, \ldots, s_{Q}\right]^{T}$ containing $Q L$-PSK symbols is dispersed according to the space-time block matrix $\mathbf{S}$ of (1) by the "ST mapper," spanning $M$ spatial and $T$ temporal dimensions. More explicitly, $\mathbf{S}$ is given by

$$
\mathbf{S}=\sum_{q=1}^{Q} \mathbf{A}_{q} s_{q}, \quad q=1, \ldots, Q
$$

where $\mathbf{A}_{q}$ having a size of $(M \times T)$ elements represents the $q$ th dispersion matrix. Hence, $\mathbf{S}$ is transmitted over the uncorrelated Rayleigh fading channel contaminated by AWGN at each receive antenna. Since unity-rate precoders are employed, a $\operatorname{PLDC}(M N T Q)$ scheme's rate is the same as that of an $\operatorname{LDC}(M N T Q)$ scheme, which is defined as $R_{\mathrm{LDC}}=Q / T$ (sym/slot).

The weighting coefficient vectors $\boldsymbol{\lambda}$ and $\gamma$ are assumed to be known at the receiver. The received signal matrix $\mathbf{Y}$ is related to $\mathrm{S}$ by

$$
\mathbf{Y}=\sqrt{\frac{\rho}{M}} \mathbf{H S}+\mathbf{V}
$$

where the channel impulse response (CIR) matrix $\mathbf{H}$ models an independent identically distributed flat Rayleigh fading channel. The channel's envelope and phase are assumed to be constant during $T$ symbol periods, but they are faded independently from one space-time matrix to the next. The CIR matrix $\mathbf{H}$ is assumed to be known to the receiver but not to the transmitter. The noise matrix $\mathbf{V}$ is assumed to have independent samples of a zero-mean unit-variance complex-valued Gaussian random process, and $\rho$ denotes the SNR.

It is desirable to rewrite the input-output matrix relationship of (2) in an equivalent vectorial form. Define the vec() operation as the vertical stacking of the columns of an arbitrary matrix. Subjecting both sides of (2) to the vec() operation gives the equivalent system matrix [10]

$$
\overline{\mathbf{Y}}=\sqrt{\frac{\rho}{M}} \overline{\mathbf{H}} \chi \mathbf{K}+\overline{\mathbf{V}}
$$

where $\overline{\mathbf{Y}} \in \zeta^{N T \times 1}$ denotes the equivalent matrix of complexvalued received signals, and $\overline{\mathbf{V}} \in \zeta^{N T \times 1}$ represents the corresponding complex-valued noise matrix. More explicitly, $\chi$ having a size of $(M T \times Q)$ is referred to as the dispersion character matrix (DCM), which is given by

$$
\chi=\left[\operatorname{vec}\left(\mathbf{A}_{1}\right), \operatorname{vec}\left(\mathbf{A}_{2}\right), \ldots, \operatorname{vec}\left(\mathbf{A}_{Q}\right)\right]
$$

while $\overline{\mathbf{H}}$ in (3) is given by

$$
\overline{\mathbf{H}}=\mathbf{I}_{T} \otimes \mathbf{H}
$$

where $\otimes$ denotes the Kronecker product, and $\mathbf{I}_{T}$ is the identity matrix having a size of $(T \times T)$. Note that the DCM $\chi$ uniquely and unambiguously determines a particular $\operatorname{LDC}(M N T Q)$.

Again, the IR-PLDC decoder's "irregular partitioner" in Fig. 1 determines the specific portion of the received signal, as well as the a priori information $I_{A}\left(u_{2}\right)$ to be detected by each PLDC component decoder, according to the weighting coefficient vector $\boldsymbol{\lambda}$. Similarly, the IRCC decoder's "irregular partitioner" operates according to the weighting coefficient vector $\bar{\gamma}=\left[\bar{\gamma}_{1}, \ldots, \bar{\gamma}_{P_{\text {out }}}\right]$. Note that the IRCC encoder's weighting coefficient vector $\gamma$ quantifies the specific fraction of input bits encoded by each component at the transmitter side, whereas $\bar{\gamma}$ determines the fraction of the log-likelihood ratios (LLRs) fed into each individual decoder at the IRCC's decoder, and the components of $\gamma$ and $\bar{\gamma}$ are related by

$$
\gamma_{i}=\frac{\bar{\gamma}_{i} \times R_{i, \mathrm{IRCC}}}{R_{\text {out }}}, \quad i=1, \ldots, P_{\text {out }} .
$$

Then, an iterative decoding structure is employed, where extrinsic information is exchanged between the three soft-insoft-out modules, namely, the MMSE detector [24], the precoder, and the outer IRCC decoder, in a number of consecutive iterations. To be specific, in Fig. 1, $I_{A}()$ denotes the a priori information represented in terms of LLRs, while $I_{E}()$ denotes the extrinsic information also expressed in terms of LLRs. Note that the intermediate rate-1 precoder processes two a priori inputs, namely, those arriving from the MMSE detector and those from the outer decoder, and generates two extrinsic outputs. More detailed discussions on the iterative decoding process are provided in [15]. It is worth mentioning that the activation of different IR-PLDC components is implemented by employing different dispersion matrices, and the associated hardware cost is modest, since it does not require the implementation of $P$ separate component codes at the transmitter and receiver. By contrast, the component codes of the IRCC scheme are obtained by puncturing of and/or adding extra generator polynomials to a selected mother RSC code; hence, the associated complexity may become potentially high.

\section{EXIT-CHART-BASED DESIGN OF IRCC-CODED IR-PLDCS}

In our forthcoming EXIT chart analysis, the unity-rate precoder's decoder and the MMSE decoder are considered as a single "inner" decoding block, constituted by the IR-PLDC's decoder seen in Fig. 1. The advantage of this representation is that the IR-PLDC block's extrinsic information output $I_{E}\left(u_{2}\right)$ is entirely determined by the received signal matrix $\mathbf{Y}$ and the a priori input $I_{A}\left(u_{2}\right)$; hence, it remains unaffected by the 
extrinsic information exchange between the precoder's decoder and the MMSE detector. Thus, we can project the three-stage system into a two-stage system, and hence, the traditional 2-D EXIT charts [23], [25] are applicable.

Following the approach of [26], we now carry out the EXIT chart analysis of the proposed IR-PLDC scheme. Thus, the corresponding EXIT transfer function is

$$
I_{E}\left(u_{2}\right)=\Gamma_{\mathrm{in}}\left[I_{A}\left(u_{2}\right), \rho\right] .
$$

The employment of irregular codes was proposed by Tüchler and Hagenauer [19], [20], who used IRCCs as an outer channel code. In [19], the authors have shown that the aggregate EXIT function of an irregular code can be obtained from the linear combination of that of its component codes, under the assumption that the probability density function of the LLRs is symmetric and continuous. More explicitly, the EXIT function of the proposed inner IR-PLDC scheme is given by

$$
\Gamma_{\mathrm{in}}=\sum_{i=1}^{P_{\mathrm{in}}} \lambda_{i} \Gamma_{i}\left(I_{\mathrm{in}}, \rho\right)
$$

where $\Gamma_{i}$ denotes the EXIT function of the $i$ th PLDC component. Similarly, the EXIT function of the outer IRCC scheme becomes

$$
\Gamma_{\text {out }}=\sum_{i=1}^{P_{\text {out }}} \bar{\gamma}_{i} \bar{\Gamma}_{i}\left(I_{\text {in }}\right)
$$

where $\bar{\Gamma}_{i}$ denotes the EXIT function of the $i$ th IRCC component, and it is independent of the operating $\operatorname{SNR} \rho$.

In the following sections, we will characterize each block of the IRCC-coded IR-PLDC scheme in Fig. 1 using various parameters, which are optimized from a capacity maximization perspective with the aid of EXIT charts.

\section{A. Generating Component Codes for the IR-PLDC}

In this section, we demonstrate how to generate an inner IR-PLDC coding scheme containing $P_{\text {in }}=11$ components for a MIMO configuration having $M=2$ transmit and $N=2$ receive antennas when quadrature phase shift keying (QPSK) modulation was employed. It has been shown in [10] that the maximum achievable spatial diversity order of an $\operatorname{LDC}(M N T Q)$ is $N \cdot \min (M, T)$. We commence by setting $T$ equal to $M$ for the first component. Hence, the resultant scheme has the potential of achieving the maximum diversity order of $D=4$. By setting $Q=1$, we create an $\operatorname{LDC}(2221)$ scheme that is suitable for this parameter combination. Hence, we are able to search for the specific DCM $\chi$ that maximizes the DCMC capacity of the LDC(2221) scheme using [27]

$$
\begin{gathered}
C_{\mathrm{LDC}}^{M L}=\frac{1}{T} \max _{p\left(K_{1}\right), \ldots, p\left(K_{F}\right)} \sum_{f=1}^{F} \int_{-\infty}^{\infty} \ldots \int_{-\infty}^{\infty} p\left(\overline{\mathbf{Y}} \mid K_{f}\right) p\left(K_{f}\right) \\
\cdot \log _{2}\left(\frac{p\left(\overline{\mathbf{Y}} \mid K_{f}\right)}{\sum_{g=1}^{F} p\left(\overline{\mathbf{Y}} \mid K_{g}\right) p\left(K_{g}\right)}\right) d \overline{\mathbf{Y}} \quad \text { bits/sym } / \mathrm{Hz}
\end{gathered}
$$

TABLE I

$P_{\text {in }}=11$ COMPonent Codes OF THE IR-PLDC SCHEME IN Fig. 1 GENERATED FOR A MIMO SYSTEM HAVING $\mathbf{M}=\mathbf{2}$ AND $\mathbf{N}=\mathbf{2}$ RECEIVE ANTENNAS AND EMPLOYING QPSK MODULATION

\begin{tabular}{|c|c|c|c|c|c|c|c|c|}
\hline Index & $M$ & $N$ & $T$ & $Q$ & Rate & $D$ & Inner It. $j$ & Compl. \\
\hline 0 & 2 & 2 & 2 & 1 & 0.5 & 4 & 0 & 1571 \\
1 & $\vdots$ & $\vdots$ & $\vdots$ & 2 & 1 & 4 & 1 & 4086 \\
2 & $\vdots$ & $\vdots$ & $\vdots$ & 3 & 1.5 & 4 & 1 & 5030 \\
3 & $\vdots$ & $\vdots$ & $\vdots$ & 4 & 2 & 4 & 1 & 5974 \\
\hline 4 & $\vdots$ & $\vdots$ & 3 & 1 & 0.33 & 4 & 0 & 3285 \\
5 & $\vdots$ & $\vdots$ & $\vdots$ & 2 & 0.67 & 4 & 1 & 8562 \\
6 & $\vdots$ & $\vdots$ & $\vdots$ & 4 & 1.33 & 4 & 1 & 12546 \\
7 & $\vdots$ & $\vdots$ & $\vdots$ & 5 & 1.67 & 4 & 1 & 14538 \\
\hline 8 & $\vdots$ & $\vdots$ & 4 & 1 & 0.25 & 4 & 0 & 5639 \\
9 & $\vdots$ & $\vdots$ & $\vdots$ & 3 & 0.75 & 4 & 1 & 18126 \\
10 & $\vdots$ & $\vdots$ & $\vdots$ & 5 & 1.25 & 4 & 1 & 24974 \\
\hline
\end{tabular}

where $K_{f}$ denotes a candidate vector. Since each transmitted symbol vector contains $Q$ symbols of an $L$-PSK constellation, we have a total of $F=L^{Q}$ number of possibilities. The normalization factor $1 / T$ corresponds to the fact that an LDC scheme spans $T$ channel uses.

Consequently, we can obtain more components by gradually increasing the value of $Q$ to increase the rate. We impose the limit of $Q \leq M T$ for the sake of maintaining a low complexity, although employing a higher value of $Q$ is feasible. Hence, by increasing the value of $T$ and maximizing the corresponding DCMC capacity of each $\operatorname{LDC}(M N T Q)$, we can generate a set of beneficial LDCs. Naturally, low $Q$ and $T$ values are desirable for the sake of maintaining a low complexity. The resultant $P_{\text {in }}=11$ component codes designed for our IR-PLDC scheme are listed in Table I. Hence, an inner $\operatorname{PLDC}(M N T Q)$ scheme can be directly obtained by combining a memory-1 unity-rate precoder [21] with an LDC having the parameter (MNTQ).

The complexity of each PLDC component code is jointly determined by the precoder's memory, the MMSE detector's complexity, and the number of inner iterations $j$. To quantify the complexity in a unified manner, we count the number of addition and multiplication operations required to calculate a single LLR value in the logarithmic domain. Since the number of addition and multiplication operations can be quantified in terms of the so-called add-compare-select (ACS) arithmetic operations, the complexity of each PLDC component is quantified by the ACS operations per LLR computation. Observe in Table I that when the value of $T$ is fixed, the complexity is increased by increasing the value of $Q$. Furthermore, increasing the value of $T$ typically resulted in substantially increased complexity.

To elaborate further on the LDC's ability to support arbitrary $(M N T Q)$ parameter combinations, as well as support arbitrary modulation schemes, in Table II, we characterized another group of LDCs designed for a MIMO system having $M=3$ and $N=2$ antennas and using binary phase shift 
TABLE II

$P_{\text {in }}=6$ COMPONENT CODES OF THE IR-PLDC SCHEME IN Fig. 1 GENERATED FOR A MIMO SYSTEM HAVING $\mathbf{M}=\mathbf{3}$ AND $\mathbf{N}=\mathbf{2}$ ANTENNAS AND EMPLOYING BPSK MODULATION

\begin{tabular}{|c|c|c|c|c|c|c|c|c|}
\hline Index & $M$ & $N$ & $T$ & $Q$ & Rate & $D$ & Inner It. $j$ & Compl. \\
\hline 0 & 3 & 2 & 3 & 1 & 0.33 & 6 & 0 & 2399 \\
1 & $\vdots$ & $\vdots$ & $\vdots$ & 2 & 0.67 & 6 & 1 & 6694 \\
2 & $\vdots$ & $\vdots$ & $\vdots$ & 3 & 1 & 6 & 1 & 8590 \\
3 & $\vdots$ & $\vdots$ & $\vdots$ & 4 & 1.33 & 6 & 1 & 10486 \\
4 & $\vdots$ & $\vdots$ & $\vdots$ & 5 & 1.67 & 6 & 1 & 12382 \\
5 & $\vdots$ & $\vdots$ & $\vdots$ & 6 & 2 & 6 & 1 & 14278 \\
\hline
\end{tabular}

keying (BPSK) modulation. Again, we set $T=3$ to achieve the maximum possible diversity gain at a low decoding complexity. It is certainly feasible to choose higher $T$ values to increase the integrity at the price of higher complexity.

\section{B. Number of Inner Iterations $j$}

The number of "inner" iterations between the MMSE detector and the precoder required for each PLDC component in Table I is also optimized for the sake of achieving the maximum capacity. In other words, we propose to answer the question of how many inner iterations $(j)$ per outer iteration are necessary for approaching the capacity. Ideally, a small value of $j$ is desirable in the interest of minimizing the decoding complexity.

The so-called area property [25], [28] of EXIT charts may be formulated by stating that the area under the "outer" IRCC curve is approximately equal to its code rate $R_{\text {out }}$. Thus, if we assume that the area under the EXIT curve of an outer code can be perfectly matched to the area under the inner code's EXIT curve at any SNR, then it is possible to approximate the maximum achievable rate of a serial concatenated scheme by evaluating the area under the EXIT curves, given the rate of the "inner" IR-PLDC code $R_{\text {in }}$, which is expressed as

$$
C(\rho)=\log _{2}(L) \cdot R_{\text {in }} \cdot R_{\text {out }} \quad \text { bits } / \mathrm{sym} / \mathrm{Hz}
$$

when $L$-PSK modulation is used.

Fig. 2 quantifies the maximum achievable rates for the different PLDC component codes in Table I employing different number of inner iterations $j$. For each set of comparisons, the DCMC capacity of the LDC using (10) is plotted as a benchmark. For the rate $R_{3,2224}=2 \operatorname{PLDC}(2224)$ scheme, we observe a clear gap between the DCMC capacity and the corresponding maximum rate, when the number of inner iterations is $j=0$. However, when we have $j=1$, the aforementioned rate loss is eliminated, and a further increase of the number of inner iterations $j$ has only a modest additional rate improvement. In fact, the maximum achievable rate loss is less than $1 \%$, when we have $j=1$. For the PLDC(2222) scheme in Table I having a rate of $R_{1,2222}=1$, we observe in Fig. 2 that although the aforementioned maximum achievable rate loss compared with the DCMC capacity is still present, when employing $j=0$ inner iterations, the associated discrepancy is narrower than that seen for the PLDC(2224) scheme. Observe in Fig. 2 for the

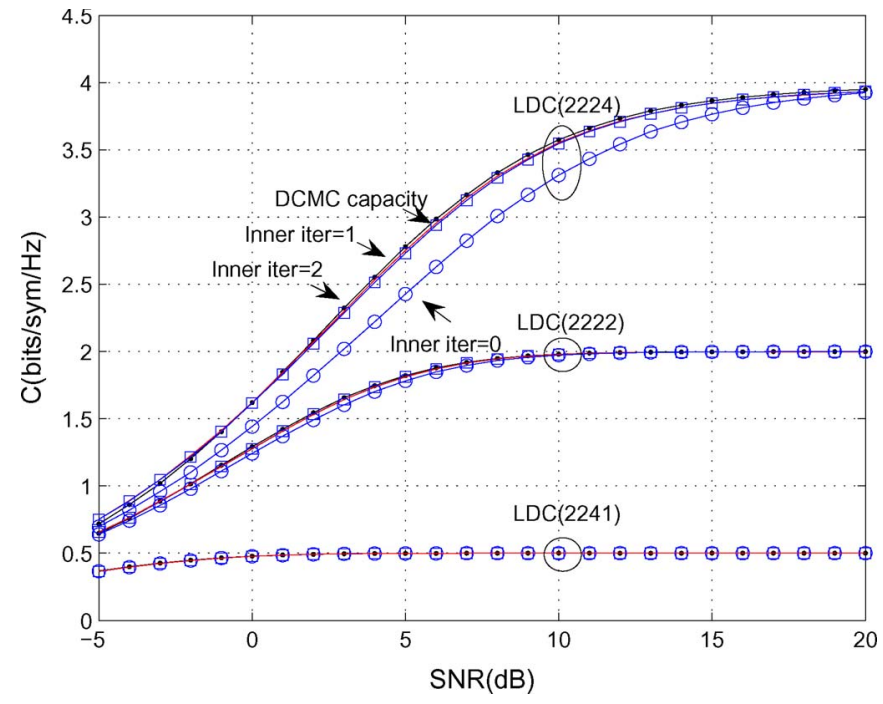

Fig. 2. Comparison of the maximum achievable rates for three PLDC schemes in Table I having $j=0,1$, and 2 inner iterations when using QPSK modulation in conjunction with an MMSE detector.

PLDC(2241) scheme having a rate of $R_{8,2241}=0.25$ that there is no maximum achievable rate loss even in the absence of inner iterations.

The above observations are related to the EXIT characteristics of the LDC MMSE decoding block. When a single symbol is transmitted, the resultant EXIT curve is a horizontal line, which is a property of the Gray labeling employed [16]. Therefore, regardless of the number of inner iterations employed, the MMSE detector in Fig. 1 always outputs the same extrinsic information. When $Q$ is increased, the resultant EXIT curve becomes more steep; therefore, higher extrinsic information can be obtained upon increasing the a priori information by using a higher number of inner iterations. Therefore, the resultant maximum achievable rate observed in Fig. 2 has an increasing discrepancy with respect to the DCMC capacity, when a higher number of symbols $Q$ is transmitted by each LDC block. We observe that for the component PLDCs in Table I, where we have $Q>1$, employing $j=1$ inner iteration will enable the system to attain $99 \%$ of the DCMC capacity. Similarly, we are capable of determining the number of necessary inner iterations required for the group of LDCs listed in Table II designed for a MIMO system having $M=3$ antennas. Again, observe in Table II that employing a single inner iteration is adequate for the LDC schemes having $Q>1$ to approach the associated DCMC capacity.

\section{Optimizing the Weighing Coefficient Vector}

Apart from the specific shape of the component EXIT curves, the aggregate inner IR-PLDC and outer IRCC schemes' EXIT curves characterized in (8) and (9) are also affected by the weighting coefficient vectors $\lambda$ and $\bar{\gamma}$. Since $\lambda$ quantifies the specific fraction of information bits fed into the IR-PLDC encoder/decoder in Fig. $1, \boldsymbol{\lambda}=\left[\lambda_{1}, \ldots, \lambda_{P_{\text {in }}}\right]$ has to satisfy

$$
1=\sum_{i=1}^{P_{\text {in }}} \lambda_{i}, \quad \lambda_{i} \in[0,1] .
$$


Similarly, the IRCC's weighting coefficient vector $\bar{\gamma}$ quantifying the fraction of the LLRs $I_{A}\left(c_{1}\right)$ fed into the IRCC decoder has to obey

$$
1=\sum_{i=1}^{P_{\text {out }}} \overline{\gamma_{i}}, \quad \overline{\gamma_{i}} \in[0,1]
$$

and the resultant aggregate rates $R_{\text {in }}$ and $R_{\text {out }}$ are given as follows:

$$
\left\{\begin{array}{l}
1 / R_{\mathrm{in}}=\sum_{i=1}^{P_{\mathrm{in}}} \frac{1}{R_{(i, \mathrm{LDC})}} \cdot \lambda_{i} \\
R_{\text {out }}=\sum_{i=1}^{P_{\text {out }}} R_{(i, \mathrm{IRCC})} \cdot \bar{\gamma}_{i}
\end{array}\right.
$$

where unity-rate precoders are employed. More explicitly, given the total number of symbols $\mathbf{Q}$ transmitted using the proposed IR-PLDC, the total number of time slots $\mathbf{T}$ required becomes $\mathbf{T}=\left(\lambda_{1} \mathbf{Q} / Q_{1}\right) T_{1}+\cdots+\left(\lambda_{P_{\text {in }}} \mathbf{Q} / Q_{P_{\text {in }}}\right) T_{P_{\text {in }}}$. Therefore, we can derive the aggregate inner rate $R_{\text {in }}$ from the rate $R_{i, \mathrm{LDC}}$ of its components as follows:

$$
\begin{aligned}
\frac{1}{R_{\mathrm{in}}} & =\frac{\mathbf{T}}{\mathbf{Q}}=\frac{\lambda_{1}}{Q_{1}} T_{1}+\cdots+\frac{\lambda_{P_{\mathrm{in}}}}{Q_{P_{\mathrm{in}}}} T_{P_{\mathrm{in}}} \\
& =\frac{\lambda_{1}}{R_{1, \mathrm{LDC}}}+\cdots+\frac{\lambda_{P_{\mathrm{in}}}}{R_{P_{\mathrm{in}}, \mathrm{LDC}}} .
\end{aligned}
$$

Our design objective is to achieve the maximum throughput $C(\rho)$ at a specific SNR $\rho$, while maintaining a vanishing BER. Hence, we conducted an exhaustive search for all the possible combinations of $\bar{\gamma}$ and $\boldsymbol{\lambda}$ under the following constraints.

1) An open convergence tunnel must exist between the inner and outer EXIT curves quantified in (8) and (9) to achieve a vanishing BER, provided that the aid of the decoding trajectory arrives at the top right corner of the EXIT chart.

2) The throughput of (11) has to be maximized.

3) The resultant open EXIT tunnel area has to be maximized, for the sake of minimizing the number iterations required.

4) Weighting coefficient vectors $\lambda$ and $\bar{\gamma}$ have to obey (12) and (13), respectively.

In our investigations, we found that a weighting factor search step-size of 0.05 applied across the entire space was sufficient to explore the benefits of irregularity in the proposed system.

The reason that our IRCC-coded IR-PLDC scheme is seeking the specific solution maximizing the open tunnel area is justified as follows. ${ }^{1}$ The rationale of employing irregular inner and/or outer codes for an iteratively detected scheme is to maximize the achievable rate. When using IRCCs as an outer code, minimizing the EXIT tunnel area corresponds to maximizing the achievable rate, owing to the EXIT chart's "area property" [25]. In other words, IRCCs aim to find an outer EXIT curve that "matches" a given inner EXIT curve as closely as possible by maximizing the area under the IRCC's EXIT curve, since this maximizes its rate. By contrast, there is no one-to-one

\footnotetext{
${ }^{1}$ The authors are grateful to the anonymous reviewer for pointing out that other techniques for identifying attractive IRCCs/IR-PLDCs can also be used, although they are expected to impose a higher weighting coefficient optimization procedure.
}

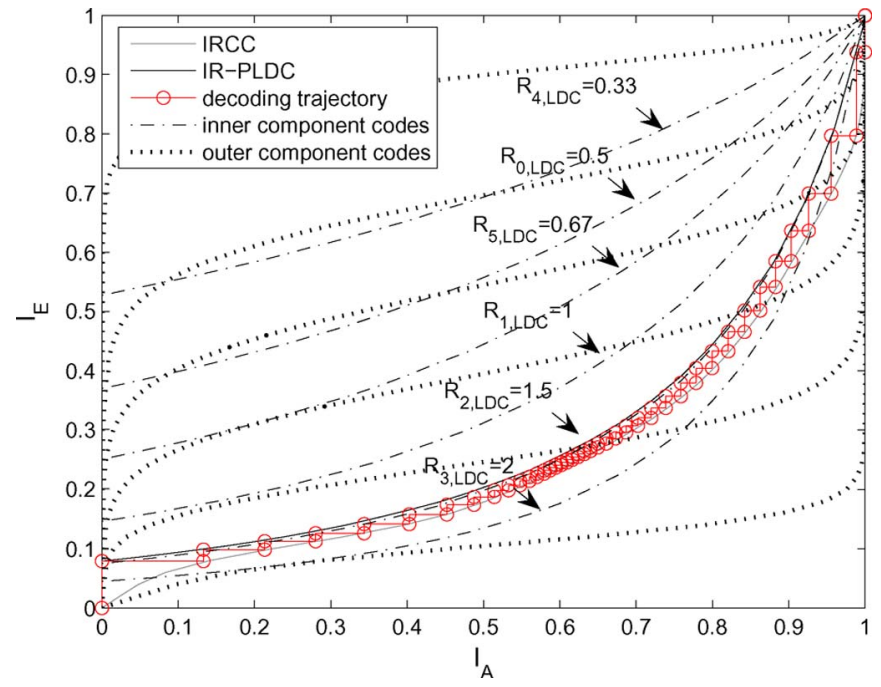

Fig. 3. EXIT chart and the decoding trajectory of the IRCC-coded IR-PLDC scheme in Fig. 1 recorded at $\rho=-4 \mathrm{~dB}$ using the component codes in Table I when employing QPSK modulation and an MMSE detector.

relationship between $R_{\text {in }}$ of the IR-PLDC and the area under its EXIT curves. Consequently, the IR-PLDC scheme offers multiple area values under its EXIT curves for any given rate $R_{\text {in }}$. Having a larger EXIT tunnel area potentially requires less iterations to achieve a vanishing BER. Therefore, our design criterion when employing an IR-PLDC as the inner code is to maximize the achievable rate $C(\rho)$ of $(11)$, while maintaining an open EXIT tunnel. If identical maximum rates are achieved by using different weighting coefficient vectors, additionally, the associated open EXIT tunnel area is also maximized.

\section{Simulation Results}

In this section, we present our numerical results characterizing the proposed IRCC-coded IR-PLDC scheme in Fig. 1 designed to operate near the MIMO channel's capacity, while maintaining a vanishing BER. In all of our simulations, the interleavers in Fig. 1 were set to have a length of $10^{6}$ bits. We employed a total of $P=12$ component codes, where the IRPLDC scheme contains the $P_{\text {in }}$ number of component codes in Tables I or II, according to the specific MIMO system. The IRCC scheme employs $P_{\text {out }}=6$ component codes having a rate of $R_{i, \mathrm{IRCC}}=[0.1,0.25,0.4,0.55,0.7,0.9]$.

Fig. 3 presents both the resultant EXIT chart and the corresponding decoding trajectory of our IRCC-coded IR-PLDC scheme designed for operating at $\rho=-4 \mathrm{~dB}$ when employing QPSK modulation combined with an MMSE detector. The dotted lines denote the EXIT curves recorded for the aforementioned set of outer IRCC components, while the area under the curves is proportional to their code rates [20]. The dasheddotted curves represent the inner EXIT curves of the first six PLDC component codes in Table I. Observe that the area under the inner component EXIT curves is increased when the rate is decreased, which corresponds to the different component PLDCs having different maximum achievable rates. The shape of all the dashed-dotted curves is similar to each other, since they are all combined with the same memory-1 precoder, 


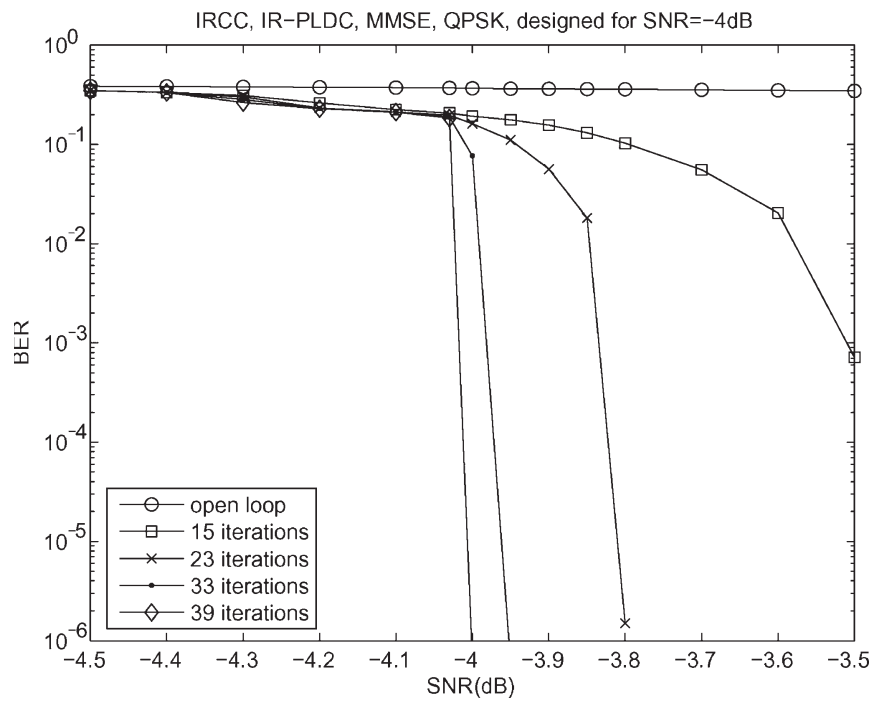

Fig. 4. BER of the IRCC-coded IR-PLDC scheme in Fig. 1 designed to achieve a vanishing BER at $\rho=-4 \mathrm{~dB}$ using the component codes of Table I when employing QPSK modulation and an MMSE detector.

which dominates the shape of the EXIT characteristics. The solid lines represent the aggregate EXIT curves of the outer IRCC and the inner IR-PLDC having the optimized weighting coefficients of

$$
\left\{\begin{array}{l}
\bar{\gamma}=[0.5,0.25,0.1,0.05,0.1,0] \\
\lambda=[0,0,0.8,0.2,0,0]
\end{array}\right.
$$

Hence, the aggregate rates $R_{\text {in }}$ and $R_{\text {out }}$ of (14) can be obtained using the aforementioned weighting coefficients. Therefore, the resultant total effective throughput $C(\rho)$ of the system calculated according to (11) becomes $0.78945 \mathrm{bits} / \mathrm{sym} / \mathrm{Hz}$. By simultaneously maximizing $C(\rho)$ and the open EXIT tunnel area, the optimized inner and outer EXIT curves in Fig. 3 exhibit a narrow open tunnel area, where the decoding trajectory in Fig. 3 shows that $k=39$ outer iterations were required for reaching the point $(1.0,1.0)$ of perfect convergence.

The corresponding BER of the IRCC-coded IR-PLDC system designed for achieving a vanishing BER at $\rho=-4 \mathrm{~dB}$ is shown in Fig. 4. As predicted in the EXIT chart in Fig. 3, a turbo cliff is observed at $\rho=-4 \mathrm{~dB}$ in Fig. 4 when $k=39$ outer iterations were carried out between the IRCC and the IRPLDC decoders.

Naturally, the flexibility of the proposed system in Fig. 1 provided by the embedded irregularity allows the system to achieve a high rate, while maintaining a vanishing BER, although at the expense of an excessive number of iterations. To quantify the relationship between the degree of irregularity and the associated maximum rates, we compare the maximum rates of four irregular systems, where different degrees of irregularity were imposed at the outer and the inner codes, while having a total number of $P=12$ component codes. More explicitly, the four systems are described as follows.

- Scheme 1: This is the IRCC-coded PLDC(2224) scheme, where we have $P_{\text {out }}=11$ and $P_{\text {in }}=1$. The PLDC(2224) scheme in Table I is the only component code of the IR-PLDC.

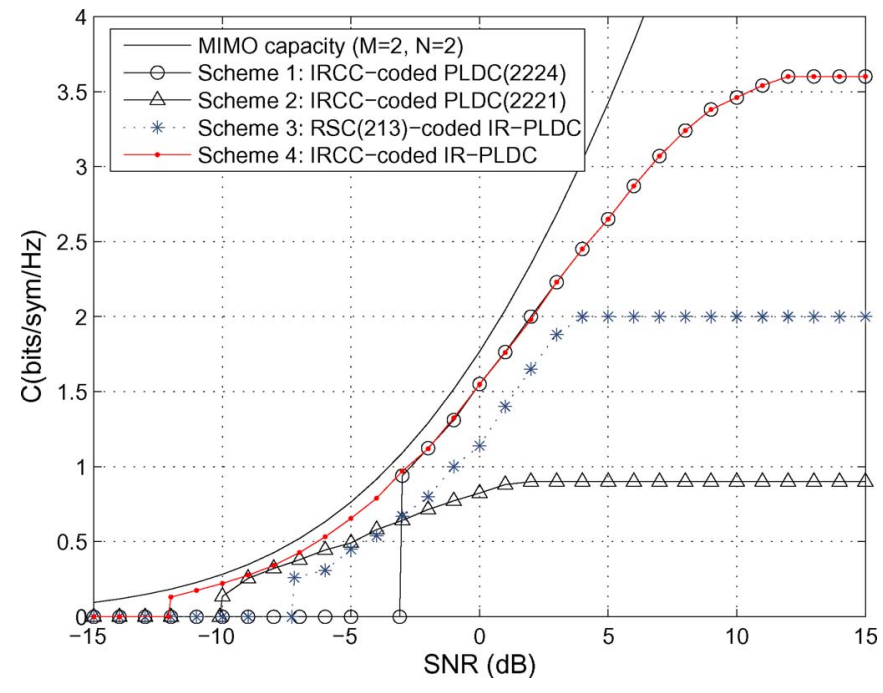

Fig. 5. Maximum rates achieved by the $P=12$ components IRCC-coded IR-PLDC scheme in Fig. 1 having different irregularity distributions and employing the component codes in Table I when QPSK modulation combined with an MMSE detector was employed.

- Scheme 2: This is the IRCC-coded PLDC(2221) scheme, where we have $P_{\text {out }}=11$ and $P_{\text {in }}=1$. The PLDC(2221) arrangement in Table I is the only component code of the IR-PLDC.

- Scheme 3: This is the RSC(213)-coded IR-PLDC scheme, where we have $P_{\text {out }}=1$ and $P_{\text {in }}=11$. A regular RSC(213) code is employed as the outer code, and the inner IR-PLDC scheme contains the first $P_{\text {in }}=11$ component codes in Table I.

- Scheme 4: This is the IRCC-coded IR-PLDC scheme as illustrated in Figs. 3 and 4, where we have $P_{\text {out }}=6$ and $P_{\text {in }}=6$.

The rate of the $P_{\text {out }}=11$ IRCC component codes is given by $R_{i, \mathrm{IRCC}}=[0.1,0.2,0.3,0.35,0.4,0.5,0.6,0.7,0.75,0.8,0.9]$.

Fig. 5 explicitly plots the maximum rates achieved by the aforementioned four irregular schemes when QPSK modulation combined with an MMSE detector was employed. Each curve in Fig. 5 was designed to achieve the maximum rate with the aid of the specific weighting coefficient vectors $\lambda$ and $\bar{\gamma}$. We observe in Fig. 5 that the IRCC-coded IR-PLDC Scheme 4 is capable of maintaining a vanishing BER for SNRs in excess of $\rho=-12 \mathrm{~dB}$, while operating as close as about $0.9 \mathrm{~dB}$ from the MIMO channel's capacity [2]. The proposed IR-PLDC scheme results in the well-understood turbo-cliff effect at a certain SNR, below which no open EXIT tunnel can be formed, hence degrading the achievable performance. By contrast, above this value, the Monte-Carlo-based simulation becomes capable of reaching the top right corner of the EXIT chart, where a vanishing BER is achieved. In addition, note that when we have $\rho>2 \mathrm{~dB}$, the proposed scheme starts to gradually deviate from the MIMO channel's capacity, owing to the lack of high-rate IR-PLDC components. Hence, when adopting additional highrate PLDC components, the distance observed from the MIMO capacity in the high-SNR region can be eliminated.

For the RSC(213)-coded IR-PLDC Scheme 3, where the irregularity was purely imposed on the inner code, we observe 


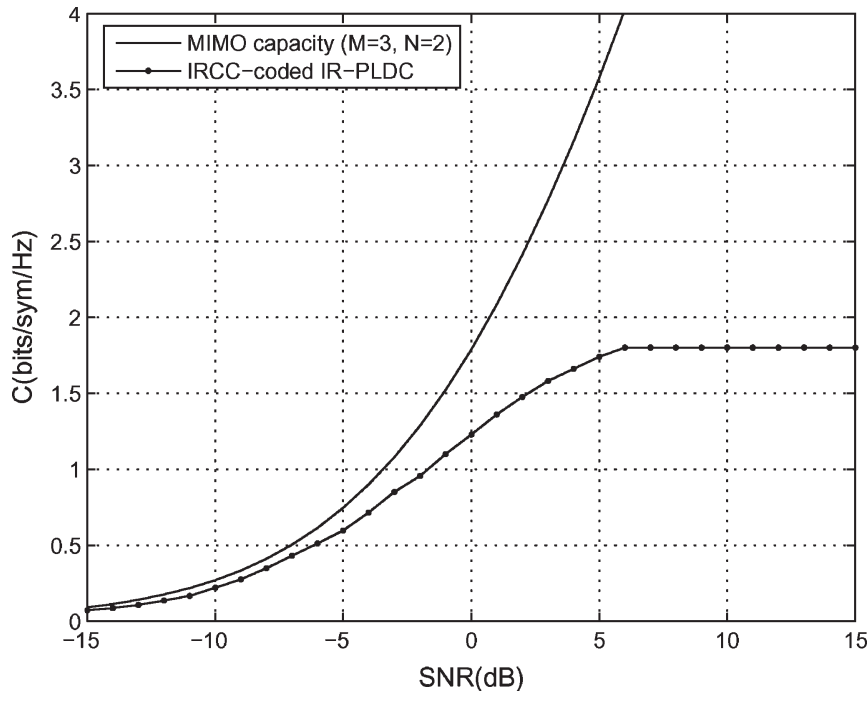

Fig. 6. Maximum rates achieved by the irregular scheme in Fig. 1 using the $P_{\text {in }}=6$ IR-PLDC scheme in Table II and the IRCCs having $P_{\text {out }}=6$ number of components when BPSK modulation combined with an MMSE detector was employed.

in Fig. 5 that it is capable of operating at approximately $2.5 \mathrm{~dB}$ from the MIMO channel's capacity. The maximum rate is limited to $2 \mathrm{bits} / \mathrm{sym} / \mathrm{Hz}$, owing to the employment of the regular half-rate $\operatorname{RSC}(213)$ code. The irregularity of the inner IR-PLDC allows the system to adequately function for SNRs spanning from $\rho=-7 \mathrm{~dB}$ to $\rho=4 \mathrm{~dB}$.

As for the IRCC-coded PLDC Schemes 1 and 2, where the irregularity was entirely assigned to the outer code, the maximum rate was essentially predetermined by the specific inner PLDC scheme employed. More explicitly, observe in Fig. 5 that when the high-rate PLDC(2224) scheme was employed, a vanishing BER was achievable for SNRs in excess of $\rho=$ $-3 \mathrm{~dB}$. Although this scheme operates at about $0.9 \mathrm{~dB}$ from the MIMO channel's capacity, it fails to function adequately in the low SNR region. By contrast, when we use the PLDC(2221) arrangement as our inner code, the system performs adequately for SNRs in excess of $\rho=-10 \mathrm{~dB}$, as seen in Fig. 5. However, this scheme suffers from a rate loss in the high-SNR region, because the inner PLDC(2221) code fails to operate near the MIMO capacity.

In summary, observe in Fig. 5 that the irregular outer schemes facilitate operation about $0.9 \mathrm{~dB}$ from the MIMO channel's capacity for SNRs either $-3 \mathrm{~dB}<\rho<6 \mathrm{~dB}$ or $-10 \mathrm{~dB}<\rho<$ $-6 \mathrm{~dB}$. By contrast, the irregular inner scheme operates across a wider SNR region at the price of having an SNR distance of about $2.5 \mathrm{~dB}$ from the MIMO capacity. Finally, when using $P_{\text {out }}=P_{\text {in }}=6$ irregular inner and outer codes, the proposed IRCC-coded IR-PLDC Scheme 4 is capable of operating at $0.9 \mathrm{~dB}$ from the MIMO capacity for SNRs $-12 \mathrm{~dB}<\rho<6 \mathrm{~dB}$.

In Fig. 6, we characterize the effective throughput achieved by the proposed IRCC-coded IR-PLDC scheme in Fig. 1 when using BPSK modulation and an MMSE detector. Note in Fig. 6 that we employ the $P_{\text {in }}=6$ number of inner component codes in Table II designed for a MIMO system having $M=3$ antennas in contrast to the $M=2$ antennas used in Fig. 5. As the outer code, we employ the same group of $P_{\text {out }}=6$
IRCC components as in Fig. 5. Observe in Fig. 6 that the irregular scheme in Fig. 1 is capable of operating close to the corresponding MIMO channel's capacity while maintaining a vanishing BER. Again, the throughput gap experienced at relatively high SNRs can be closed if high-rate inner component codes are employed.

\section{CONCLUSiON}

In this paper, we have proposed a novel IRCC-coded IR-PLDC scheme and demonstrated that it is capable of operating at about $0.9 \mathrm{~dB}$ from the MIMO channel's capacity at a potentially high complexity. Furthermore, we have shown that the proposed irregular scheme becomes capable of reliably operating for SNRs in excess of $-12 \mathrm{~dB}$ for the MIMO system having $M=2$ transmit and $N=2$ receive antennas while maintaining a vanishing BER. We have also proposed a novel method of optimizing the component LDCs according to their DCMC capacity. The other coding blocks in Fig. 1 and the choice of all the iterative decoding parameters were also optimized from a capacity-maximization perspective with the aid of EXIT charts. We have provided a detailed maximum-rate comparison for four irregular schemes, where a different degree of irregularity was imposed at both the inner and outer codes by adjusting the number of inner and outer codes. Finally, we have demonstrated the proposed system's flexibility in terms of accommodating diverse antenna configurations.

\section{REFERENCES}

[1] L. Hanzo, O. R. Alamri, M. El-Hajjar, and N. Wu, Near-Capacity MultiFunctional MIMO Systems: Sphere-Packing, Iterative Detection and Cooperation. Hoboken, NJ: Wiley, 2009.

[2] G. J. Foschini and M. J. Gans, "On limits of wireless communications in a fading environment when using multiple antennas," Wirel. Pers. Commun., vol. 6, no. 3, pp. 311-335, Mar. 1998.

[3] I. E. Telatar, "Capacity of multi-antenna Gaussian channels," Eur. Trans. Telecommun., vol. 10, no. 6, pp. 585-595, Nov./Dec. 1999.

[4] L. Z. Zheng and D. Tse, "Diversity and multiplexing: A fundamental tradeoff in multiple-antenna channels," IEEE Trans. Inf. Theory, vol. 49, no. 5, pp. 1073-1096, May 2003.

[5] B. Hassibi and B. M. Hochwald, "High-rate codes that are linear in space and time," IEEE Trans. Inf. Theory, vol. 48, no. 7, pp. 1804-1824, Jul. 2002.

[6] S. M. Alamouti, "A simple transmit diversity technique for wireless communications," IEEE J. Sel. Areas Commun., vol. 16, no. 8, pp. 1451-1458, Oct. 1998.

[7] P. Rabiei and N. Al-Dhahir, "A new information lossless STBC for 2 transmit antennas with reduced-complexity ML decoding," in Proc. Veh. Technol. Conf., Baltimore, MD, Sep. 2007, pp. 773-777.

[8] O. Alamri, S.-X. Ng, F. Guo, S. Zummo, and L. Hanzo, "Nonbinary LDPC-coded sphere-packed transmit diversity," IEEE Trans. Veh. Technol., vol. 57, no. 5, pp. 3200-3205, Sep. 2008.

[9] M. El-Hajjar, O. Alamri, S.-X. Ng, and L. Hanzo, "Turbo detection of precoded sphere packing modulation using four transmit antennas for differential space-time spreading," IEEE Trans. Wireless Commun., vol. 7, no. 3, pp. 943-952, Mar. 2008.

[10] R. W. Heath, Jr. and A. Paulraj, "Linear dispersion codes for MIMO systems based on frame theory," IEEE Trans. Signal Process., vol. 50, no. 10, pp. 2429-2441, Oct. 2002.

[11] R. H. Gohary and T. N. Davidson, "Design of linear dispersion codes: Asymptotic guidelines and their implementation," IEEE Trans. Wireless Commun., vol. 4, no. 6, pp. 2892-2906, Nov. 2005.

[12] V. Tarokh, N. Seshadri, and A. R. Calderbank, "Space-time codes for high data rate wireless communication: Performance criterion and code construction," IEEE Trans. Inf. Theory, vol. 44, no. 2, pp. 744-765, Mar. 1998. 
[13] J. C. Belfiore, G. Rekaya, and E. Viterbo, "The golden code: A $2 \times 2$ full-rate space-time code with nonvanishing determinants," IEEE Trans. Inf. Theory, vol. 51, no. 4, pp. 1432-1436, Apr. 2005.

[14] Y. Hong, E. Viterbo, and J. C. Belfiore, "Golden space-time trellis coded modulation," IEEE Trans. Inf. Theory, vol. 53, no. 5, pp. 1689-1705, May 2007.

[15] S. Benedetto, D. Divsalar, G. Montorsi, and F. Pollara, "Serial concatenation of interleaved codes: Performance analysis, design, and iterative decoding," IEEE Trans. Inf. Theory, vol. 44, no. 3, pp. 909-926, May 1998.

[16] O. Alamri, N. Wu, and L. Hanzo, "A differential turbo detection aided sphere packing modulated space-time coding scheme," in Proc. Veh. Technol. Conf., Melbourne, Australia, May 2006, vol. 5, pp. 2474-2478.

[17] B. M. Hochwald and S. ten Brink, "Achieving near-capacity on a multipleantenna channel," IEEE Trans. Commun., vol. 51, no. 3, pp. 389-399, Mar. 2003.

[18] Y. Zhang, "Approaching v-BLAST capacity with adaptive modulation and LDPC encoding," IEEE Trans. Commun., vol. 55, no. 12, pp. 2261-2269, Dec. 2007.

[19] M. Tüchler and J. Hagenauer, "Exit charts of irregular codes," in Proc. 36th CISS, Mar. 2002, pp. 748-753.

[20] M. Tüchler, "Design of serially concatenated systems depending on the block length," IEEE Trans. Commun., vol. 52, no. 2, pp. 209-218, Feb. 2004.

[21] D. Divsalar, S. Dolinar, and F. Pollara, "Serial concatenated trellis coded modulation with rate-1 inner code," in Proc. IEEE Global Telecommun. Conf., San Francisco, CA, Nov. 2000, vol. 2, pp. 777-782.

[22] S. ten Brink, J. Speidel, and R. H. Yan, "Iterative demapping and decoding for multilevel modulation," in Proc. IEEE Global Telecommun. Conf., Sydney, Australia, Nov. 8-12, 1998, vol. 1, pp. 579-584.

[23] S. ten Brink, "Designing iterative decoding schemes with the extrinsic information transfer chart," AEÜ Int. J. Electron. Commun., vol. 54, no. 6, pp. 389-398, 2000.

[24] S. Tan, S. Chen, and L. Hanzo, "Iterative multiuser minimum symbol error rate beamforming aided QAM receiver," IEEE Signal Process. Lett., vol. 15 , pp. 301-304, 2008

[25] A. Ashikhmin, G. Kramer, and S. ten Brink, "Extrinsic information transfer functions: Model and erasure channel properties," IEEE Trans. Inf. Theory, vol. 50, no. 11, pp. 2657-2673, Nov. 2004

[26] F. Brannstrom, L. K. Rasmussen, and A. J. Grant, "Convergence analysis and optimal scheduling for multiple concatenated codes," IEEE Trans. Inf. Theory, vol. 51, no. 9, pp. 3354-3364, Sep. 2005.

[27] S. X. Ng and L. Hanzo, "On the MIMO channel capacity of multidimensional signal sets," IEEE Trans. Veh. Technol., vol. 55, no. 2, pp. 528-536, Mar. 2006.

[28] A. Ashikhmin, G. Kramer, and S. ten Brink, "Code rate and the area under extrinsic information transfer curves," in Proc. IEEE Int. Symp. Inf. Theory, 2002, p. 115

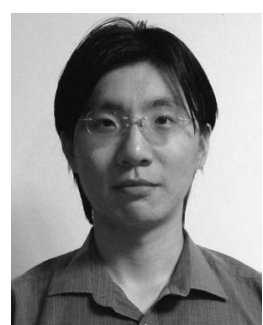

Nan Wu received the B.Eng. degree in electronics engineering from the Dalian University of Technology, Dalian, China, in 2003 and the M.Sc. (with Distinction) and $\mathrm{Ph} . \mathrm{D}$. degrees from the University of Southampton, Southampton, U.K., in 2004 and 2008, respectively.

In September 2008, he joined the National Institute of Standards and Technology, as a Guest Researcher working on cross-layer designs. His research interests are in the area of wireless communications, including space-time coding, channel coding, and cooperative multiple-input-multiple-output systems.

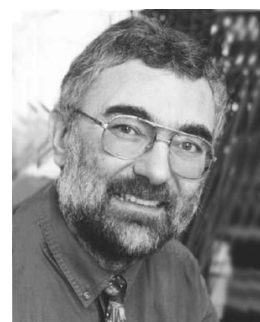

Lajos Hanzo (F'04) received the Dipl.Ing. (Masters) and Ph.D. degrees from the Technical University of Budapest, Budapest, Hungary, in 1976 and 1983, respectively, and the D.Sc. degree from the University of Southampton, Southampton, U.K., in 2004.

During his 31-year career in telecommunications, he has held various research and academic posts in Hungary, Germany, and the U.K. Since 1986, he has been with the School of Electronics and Computer Science, University of Southampton, Southampton, U.K. where he holds the position of Chair in telecommunications. Currently, he is directing an academic research team that is working on a range of research projects in the field of wireless multimedia communications sponsored by industry, the Engineering and Physical Sciences Research Council, U.K., the European IST Program, and the Mobile Virtual Centre of Excellence, U.K. He is an enthusiastic supporter of industrial and academic liaison, and he offers a range of industrial courses. He is a coauthor of 17 books on mobile radio communications, totaling more than 10000 pages. He has published more than 800 research papers and has presented keynote lectures.

Dr. Hanzo is a Fellow of the Royal Academy of Engineering and the Institution of Engineering and Technology. He has been the recipient of a number of distinctions. He is also an IEEE Distinguished Lecturer and a Governor of both the IEEE Communications and IEEE Vehicular Technology Societies. He is the acting Editor-in-Chief of the IEEE Press. He has acted as the TPC Chair of several IEEE conferences. 